University of Nebraska - Lincoln

DigitalCommons@University of Nebraska - Lincoln

$10-9-2000$

\title{
Nonadiabatic heat-capacity measurements using a superconducting quantum interference device magnetometer
}

\author{
A.I. Kharkovski \\ Laboratorium fu"r Angewandte Physik, Gerhard-Mercator-Universita"t, Duisburg, Germany \\ Christian Binek \\ University of Nebraska-Lincoln, cbinek@unl.edu \\ Wolfgang Kleeman \\ Laboratorium fu"r Angewandte Physik, Gerhard-Mercator-Universita"t, Duisburg, Germany, \\ wolfgang.kleemann@uni-due.de
}

Follow this and additional works at: https://digitalcommons.unl.edu/physicsbinek

Part of the Physics Commons

Kharkovski, A.I.; Binek, Christian; and Kleeman, Wolfgang, "Nonadiabatic heat-capacity measurements using a superconducting quantum interference device magnetometer" (2000). Christian Binek Publications. 14.

https://digitalcommons.unl.edu/physicsbinek/14

This Article is brought to you for free and open access by the Research Papers in Physics and Astronomy at DigitalCommons@University of Nebraska - Lincoln. It has been accepted for inclusion in Christian Binek Publications by an authorized administrator of DigitalCommons@University of Nebraska - Lincoln. 


\title{
Nonadiabatic heat-capacity measurements using a superconducting quantum interference device magnetometer
}

\author{
A. I. Kharkovski, ${ }^{\text {a) }}$ Ch. Binek, ${ }^{\text {b) }}$ and W. Kleemann \\ Laboratorium für Angewandte Physik, Gerhard-Mercator-Universität, D47048 Duisburg, Germany
}

(Received 23 May 2000; accepted for publication 14 August 2000)

\begin{abstract}
Nonadiabatic measurements of the heat capacity involving sample-inherent thermometry are proposed. The method is realized with superconducting quantum interference device magnetometry and applied to $\mathrm{FeBr}_{2}$ single crystals by using the magnetization for both thermometry and relaxation calorimetry. When heating with a step pulse of laser light, the magnetization relaxes on a characteristic time scale $\tau=R C$, where $C$ is the heat capacity and $R$ is the heat resistance between the sample and the bath. $R$ is independently determined from the temperature dependence of the magnetic moment measured with and without stationary light irradiation. (C) 2000 American Institute of Physics. [S0003-6951(00)02441-4]
\end{abstract}

Basically there are three different classes of the heat capacity measurement described in the literature. ${ }^{1}$ On the one hand, there is the old traditional adiabatic method, where a heat pulse $\delta Q$ causes a small temperature rise $\delta T$ in the sample. The heat capacity is then calculated according to its definition, $C=\delta Q / \delta T$. On the other hand, there are nonadiabatic methods using heat links between the sample and the heat reservoir. One of those nonadiabatic methods is, e.g., the differential thermal-analysis (DTA) technique. In a typical experiment, the measurement specimen and a reference sample of well known heat capacity are heated simultaneously. If the two samples are thermally insolated from each other, temperature deviations between the sample and its reference reflect the heat capacity variation. It can be measured with a relative accuracy of $\approx 0.02 \%$ for samples of a few milligrams. ${ }^{2}$ The second group of nonadiabatic methods are the so called "ac-temperature calorimetry", 3 and the "relaxation calorimetry." 4 In the case of ac calorimetry a sinusoidal voltage of frequency $\omega$ is applied to the heater, which gives rise to a temperature oscillation of the sample at $2 \omega$. A lock-in amplifier measures the amplitude of the temperature response which allows to determine the heat capacity. In the relaxation calorimetry the power of the heater is increased stepwise. It gives rise to an exponential temperature response towards a new equilibrium temperature involving the characteristic time constant $\tau=R C$, which is determined by the heat capacity $C$ and the heat resistance $R$.

Common to all these methods is the use of an external thermometer and, frequently, of an electric heater. This concept requires calibrations of the heat capacity of the sample holder, the thermometer, and the heater, the errors of which principally limit the accuracy of the measurement. In this letter we describe a technique of heat capacity measurements without attaching an external thermometer and an electric heater to the sample. Hence, the resolution of this method is no longer limited by parasitic contributions to the total heat capacity. The basic idea of our method is to use the sample

\footnotetext{
a) On leave from the Institute for High Pressure Physics at the Russian Academy of Sciences, Troitsk, Moscow Region, 142092, Russia.

${ }^{b)}$ Electronic mail: binek@kleemann.uni-duisburg.de
}

itself as a thermometer. In the vicinity of a temperature driven phase transition there are usually several physical properties, which are related to the order parameter and, hence, exhibit pronounced temperature dependencies. If they are accessible by precise measurements, the sample can be used as an intrinsic thermometer. In order to eliminate the electric heater we used a laser light beam with an optical output power of $P_{0} \leqslant 1 \mathrm{~mW}$ at a wavelength of $\lambda=670 \mathrm{~nm}$. The heating by optical absorption $P$ enables mechanical separation between the heat source and the sample and excludes any galvanic or inductive cross talk with the measurement process, which is of great advantage, e.g., in the case of magnetic measurements.

Our heat capacity measurements have been realized with the help of a superconducting quantum interference device (SQUID) magnetometer (Quantum Design MPMS 5S). The new method is applied to samples of the metamagnet $\mathrm{FeBr}_{2}$, which are prepared under dry helium atmosphere. Typical platelets of dimensions $3 \times 3 \times 0.3 \mathrm{~m}^{3}$ (corresponding to a mass of $12 \mathrm{mg}$ ) are obtained for heat capacity measurements in axial magnetic fields $\mu_{0} H<5 \mathrm{~T}$.

A sketch of our experimental setup is shown on the Fig. 1. The sample is located in the middle of the central pickup coil, which is connected to the SQUID sensor. The absence of any special thermal isolation as in usual standard magnetic measurements gives rise to a strong thermal coupling with the surrounding He-exchange gas. In the case of constant applied magnetic field $\mu_{0} H$ and constant temperature $T$, the magnetic moment $m$ of the sample will also remain constant. However, on heating the sample with the help of a step-like increase of the fiber-guided light power, the temperature of the sample increases exponentially according

$$
\delta T=\delta T_{0}\left[1-\exp \left(-\frac{t-t_{0}}{\tau}\right)\right]
$$

to the new higher equilibrium temperature $T+\delta T_{0}$. It is clear that as a consequence the magnetic moment of the sample will also change from $m=m_{0}$ to a new value $m_{0}$ $+\delta m$. In the case of a linear dependence $m=m(T)$ the temporal relaxation of the magnetization reads $m(t)=m_{0}$ $+\delta m\left[1-\exp \left(-\left(t-t_{0}\right) / \tau\right)\right]$ with the characteristic time con- 


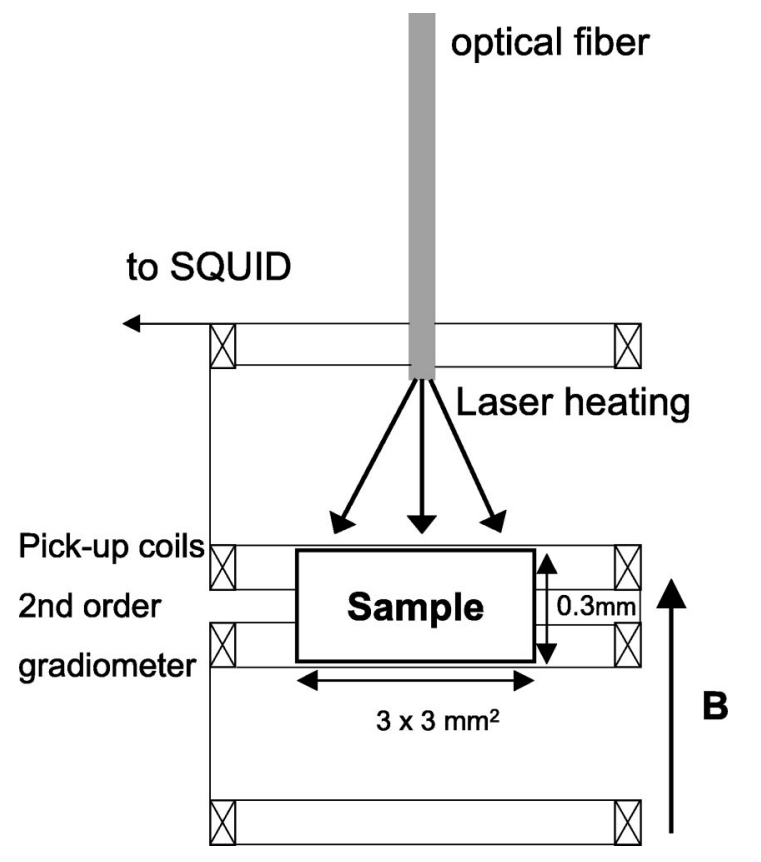

FIG. 1. Schematic sketch of the experimental setup. An optical fiber is connected with a laser diode outside of the SQUID magnetometer. The sample is mounted with the help of super glue or grease in an ordinary plastic tube on top of a small platform of low magnetic susceptibility (not shown in the figure). Pickup coils of second order gradiometer type detect the induced change of flux.

stant $\tau=R C$, where $R$ is the heat resistance and $C$ is the heat capacity, respectively. The heat resistance reflects exchange gas properties mainly and, hence, depends on temperature. The assumption of one single time constant $\tau=R C$ involved in the relaxation law, Eq. (1), was confirmed by verifying Debye-type dispersion (frequency dependence of the ac magnetic moment involving merely one relaxation time, $\tau$ ) of the magnetization response upon heating with ac power, $P_{0} \cos (\omega t){ }^{5}$ In order to determine the temperature dependence of $R$, we measured $m$ vs $T$ with and without a continuous laser light irradiation of the sample. From these two measurements the stationary temperature difference $\delta T_{0}$ can be calculated. Under the assumption of constant absorption of the laser light power $P$, the heat resistance $R=\delta T_{0} / P$ is proportional to $\delta T_{0}$ vs $T$.

A typical temperature dependence of the field induced magnetic moment, $m$, of $\mathrm{FeBr}_{2}$ is shown in Fig. 2(a) together with the corresponding $\delta T_{0}$ vs $T$ dependencies in Fig. 2(b) $\left(\mu_{0} H=2.8 \mathrm{~T}\right)$ and $\mathrm{c}\left(\mu_{0} H=2.0 \mathrm{~T}\right)$. The point of inflexion just below the peak of $m(T)$ indicates the phase transition from the paramagnetic $(\mathrm{PM})$ at $T>T_{c}\left(\mu_{0} H=2.8 \mathrm{~T}\right)=10.9 \mathrm{~K}$ to the antiferromagnetic $(\mathrm{AF})$ state $\left(T<T_{c}\right)$. The applied constant laser power has been reduced by optical filters in order to produce a sample heating of $\delta T_{0} \lesssim 0.1 \mathrm{~K}$. The ana$\log$ output of the SQUID amplifier is used to register the temporal relaxation of the magnetic moment as a reaction to a step-like onset of light power. The signal was digitized by using a 12-bit AD-converter with a temporal resolution of $1.7 \mathrm{~ms}$. Figure 3 shows three typical relaxation curves for temperatures $T$ below (AF) and above $T_{c}\left(\mu_{0} H=2.8 \mathrm{~T}\right)$ (PM). Note that according to Fig. 2(a) these relaxation curves change from positive to negative slope with increasing temperature, since $d m / d T$ vs $T$ changes its sign in the Downloaded 13 Sep 2006 to 129.93.16.206. Redistribution subject

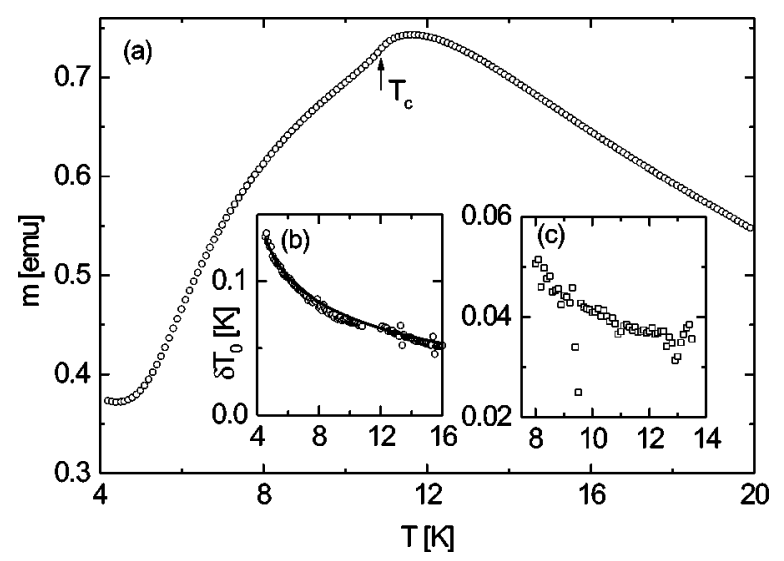

FIG. 2. (a) Temperature dependence of the magnetic moment $m$ of a $\mathrm{FeBr}_{2}$ single crystal in an axial $(H / / c)$ magnetic field $\mu_{0} H=2.8 \mathrm{~T}$. Changes of the $m$ vs $T$ dependence induced by laser light heating are not visible on this scale. Insets show the temperature dependence of the stationary light induced temperature change $\delta T_{0}$ of the sample for axial magnetic fields $\mu_{0} H=2.8 \mathrm{~T}(\mathrm{~b})$ and $\mu_{0} H=2 \mathrm{~T}(\mathrm{c})$, respectively. The $\delta T_{0}$ vs $T$ data are calculated from the $m$ vs $T$ data with and without light irradiation, respectively. Solid line (b) shows the result of a best fit of an empirical function to the data which is used for the calculation of $C$ vs $T$ for $\mu_{0} H=2.8 \mathrm{~T}$.

vicinity of $T_{c}$. It is obvious from Fig. 2(a) that the $m$ vs $T$ dependence is highly nonlinear in particular in the AF state. This must be taken into account when describing the relaxation behavior of the magnetization. We use a Taylor series expansion up to third order in $\delta T$. It reads

$m(T+\delta T) \approx m(T)+\frac{d m}{d T} \delta T+\frac{1}{2} \frac{d^{2} m}{d T^{2}} \delta T^{2}+\frac{1}{3 !} \frac{d^{3} m}{d T^{3}} \delta T^{3}$.

Inserting Eq. (1) into the series expansion yields the fitting function

$$
\begin{aligned}
m(t)= & m_{0}+A\left[1-\exp \left(-\frac{t-t_{0}}{\tau}\right)\right] \\
& +B\left[1-\exp \left(-\frac{t-t_{0}}{\tau}\right)\right]^{2} \\
& +C\left[1-\exp \left(-\frac{t-t_{0}}{\tau}\right)\right]^{3}+D t
\end{aligned}
$$

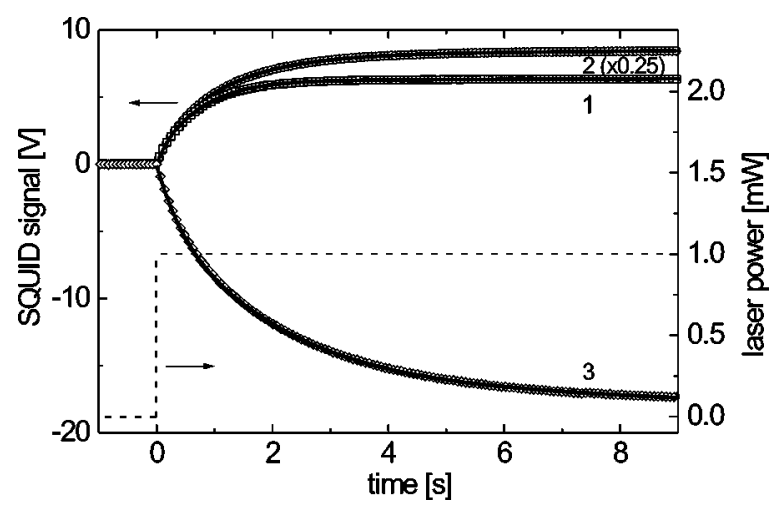

FIG. 3. Temporal relaxation curves of the magnetic moment of $\mathrm{FeBr}_{2}$ in an axial magnetic field $\mu_{0} H=2.8 \mathrm{~T}$ for $T=4.6$ (curve 1), 7 (2) and $13.8 \mathrm{~K}$ (3) on applying a step-like increase of the laser power at $t=0$ (dashed line). Curves are measured from the direct SQUID signal with the help of a 12bit analog digital converter. The lines show results of best fits of Eq. (3) to the data. 


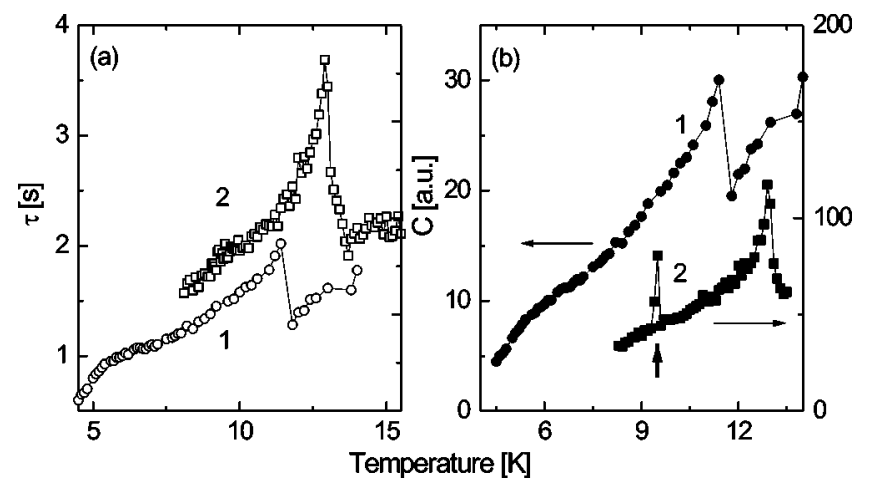

FIG. 4. (a) Temperature dependence of the characteristic $\tau$ value obtained from the best fit of Eq. (3) to the temporal relaxation data. Squares show the $\tau$ vs $T$ dependence for a $\mathrm{FeBr}_{2}$ sample in an axial field $\mu_{0} H=2 \mathrm{~T}$. Circles show the $\tau$ vs $T$ dependence for $2.8 \mathrm{~T}$ of a $\mathrm{FeBr}_{2}$ sample of about $1 / 2$ of the mass. The optical power remained constant in both measurements. (b) Corresponding temperature dependence of the heat capacity for $2 \mathrm{~T}$ (solid squares) and $2.8 \mathrm{~T}$ (solid circles) calculated from $C=\tau P / \delta T_{0}$.

where $\tau, A, B, C$, and $D$ are fitting parameters. The $D$ term reflects a linear drift of the SQUID signal, which is phenomenologically well established. Comparison of Eqs. (2) and (3) shows that the fit parameters $A, B, C$ are closely linked to the derivatives of $m(t)$. This proportionality has successfully been cross checked by numerical analysis.

Figure 4(a) shows the temperature dependencies of $\tau$ for $\mu_{0} H=2$ (curve 1) and $2.8 \mathrm{~T}$ (curve 2), which result from best fits of Eq. (3) to the relaxation data. The measurements were carried out on two different samples of $\mathrm{FeBr}_{2}$ with a mass ratio of about 2. In both cases the laser power was the same. Hence, in the case of $\mu_{0} H=2 \mathrm{~T}$, applied to the larger sample having about twofold thickness, the laser heating $\delta T_{0}$ was nearly two times smaller than in the case of $2.8 \mathrm{~T}$. It is obvious that by increasing $\delta T_{0}$ the accuracy of the determination of $\tau$ will be more accurate. However, the resolution of sharp features in the heat capacity data is limited in the case of large $\delta T_{0}$.

In order to calculate the absolute heat capacity from the $\tau$ value the simple equation $C=\tau P / \delta T_{0}$ is expected to hold. Unfortunately we do not know the absolute value of the absorbed light power $P$ for the two $\mathrm{FeBr}_{2}$ samples. Hence, Fig. 4(b) exhibits the resulting heat capacity in arbitrary units. Both curves represent the field dependent critical peaks of the magnetic phase transition from the AF into the PM state in excellent agreement with the phase diagram. ${ }^{6}$ In addition, the typical broad shoulders below $T_{c}(H)$, which originate from strong noncritical fluctuations, are shown in the $C$ vs $T$ data. ${ }^{7}$ Moreover, the $C$ vs $T$ curve for $\mu_{0} H=2 \mathrm{~T}$ even seems to reflect an additional sharp peak at $T=9.4 \mathrm{~K}$, which coincides with recent results on specific heat measurements of
Katori et al. ${ }^{8}$ The absence of such a sharp feature in the $C$ vs $T$ data at $\mu_{0} H=2.8 \mathrm{~T}$ probably originates from the smaller temperature resolution in this measurement [see Fig. 2(b)]. Surprisingly, the peak in the $2 \mathrm{~T}$ data (curve 1) is generated by a sharp minimum in the $R$ vs $T$ dependence [Fig. 2(c)], whereas no further anomaly appears in the corresponding $\tau$ vs $T$ curve.

In conclusion we have introduced a simple and universal method of heat capacity measurements by taking advantage of thermometry provided by characteristic sample properties. They are measured with one and the same apparatus. While a SQUID magnetometer was used in this presentation, similar devices can also be installed into other conventional instruments like electrometers, electrical resistance equipment, or optical setups. In principle each is able to provide thermometry by precise measurements of the temperature dependence of, e.g., the magnetization $M$, the dielectric polarization $P$, the electrical resistance $\rho$, the reflection coefficient $r$, etc. The method is completed by energy transfer excluding any cross talk with the measurement process. In our case, heating with a light beam proved to be appropriate.

We demonstrated this method with the help of a SQUID magnetometer on $\mathrm{FeBr}_{2}$ samples of a few milligrams mass. Since the determination of a magnetic phase diagram in general profits from the combined analysis of the magnetization, the susceptibility and the heat capacity of our method provides the advantage that all investigations can be performed within a single apparatus. This is important in the case of hygroscopic samples like $\mathrm{FeBr}_{2}$. Since our method of heat capacity measurements accumulates properties of the sample's environment only via the heat resistance $R$, it may easily be extended into the region of microcalorimetry, e.g., to thin film or fine particle applications, also at ultralow temperatures.

This work was supported by the Deutsche Forschungsgemeinschaft. A. K. is thankful for the hospitality experienced during his stay at the Laboratorium für Angewandte Physik of the Gerhard-Mercator Universität Duisburg.

${ }^{1}$ G. R. Stewart, Rev. Sci. Instrum. 54, 1 (1983).

${ }^{2}$ A. Schilling and O. Jeandupeux, Phys. Rev. B 52, 9714 (1995).

${ }^{3}$ P. F. Sullivan and G. Seidel, Phys. Rev. 173, 679 (1968).

${ }^{4}$ R. Bachmann, F. J. DiSalvo, Jr., T. H. Geballe, R. L. Greene, R. E. Howard, C. N. King, H. C. Kirsch, K. N. Lee, R. E. Schwall, H.-U. Thomas, and R. B. Zubeck, Rev. Sci. Instrum. 43, 205 (1972).

${ }^{5} \mathrm{Ch}$. Binek, W. Kleemann, Ch. Schwerdt, and R. Vierbaum (unpublished).

${ }^{6}$ Ch. Binek, T. Kato, W. Kleemann, O. Petracic, F. Bourdarot, P. Burlet, H. Aruga Katori, K. Katsumata, K. Prokes, and S. Welzel, Eur. Phys. J. B 15, 35 (2000).

${ }^{7}$ M. M. P. de Azevedo, Ch. Binek, J. Kushauer, W. Kleemann, and D. Bertrand, J. Magn. Magn. Mater. 140-144, 1557 (1995).

${ }^{8}$ H. Aruga Katori and K. Katsumata, Phys. Rev. B 54, R9620 (1996). 